
2018

Article DOI: https://doi.org/10.35219/im.2018.1.01

\title{
PARAMETRIC MODELING RELATED TO STEEL STRUCTURE CONSTRUCTION FOR WALL CRANE
}

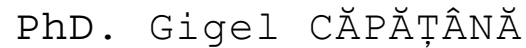 \\ PhD. Diana MUSC $\breve{A}$ (ANGHELACHE) \\ "Dunărea de Jos" University of Galați, \\ Engineering Faculty of Brăila, \\ Research Center for Mechanics of Machines \\ and Technological Equipments
}

\begin{abstract}
This paperwork develops parametric modelling of steel structure for a wall crane and jib analysis using the finite element method. Thus, we performed the modelling of a wall crane jib by using the dimensioning technological data from technical leaflets, engineering software Mechanical Desktop 2015. The analysis of stress and deformation conditions for jib was done by means of Algor V 23 software. By means of the analysis program we will find data on the crane jib behaviour under maximum load.
\end{abstract}

KEYWORDS: Finite element method, wall crane jib, stresses and deformations

\section{INTRODUCTION}

The wall crane is a rotating crane, with console, having a fixed or mobile lifting mechanism with possibility for console rotation in horizontal plane, having constant or variable outreach, depending on type and destination of this kind of crane [1], [2], [5]. group.

Wall cranes are part of slewing cranes

There are four types of slewing cranes, namely: slewing crane with pillar, wall crane, hinged slewing crane and slewing special crane. These are shown in figure 1.

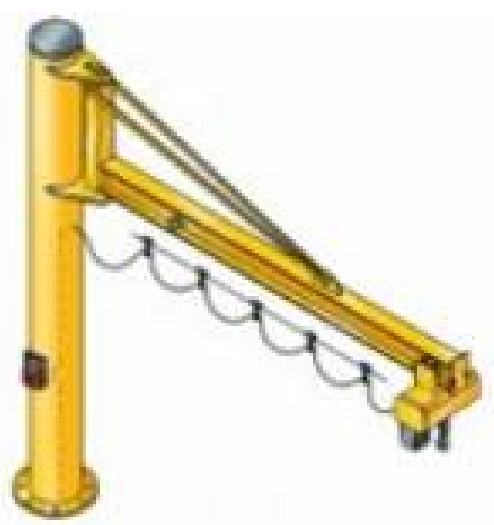

a) Slewing crane with pillar

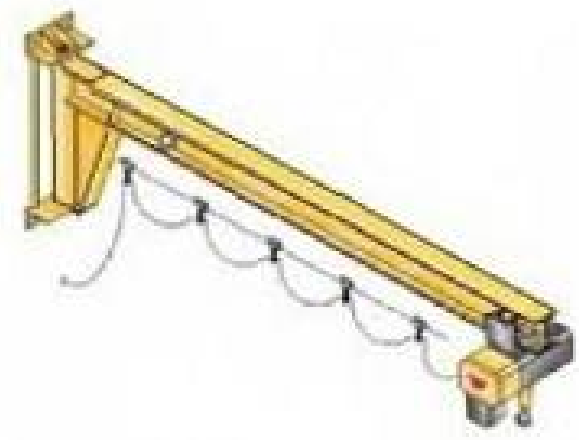

b) Wall crane

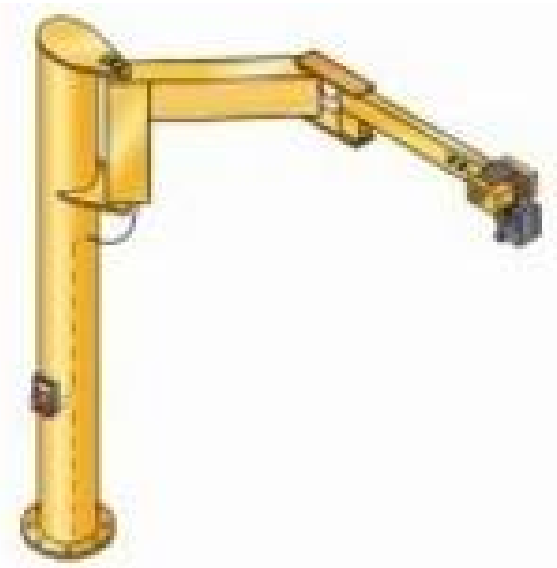


c) Hinged slewing crane

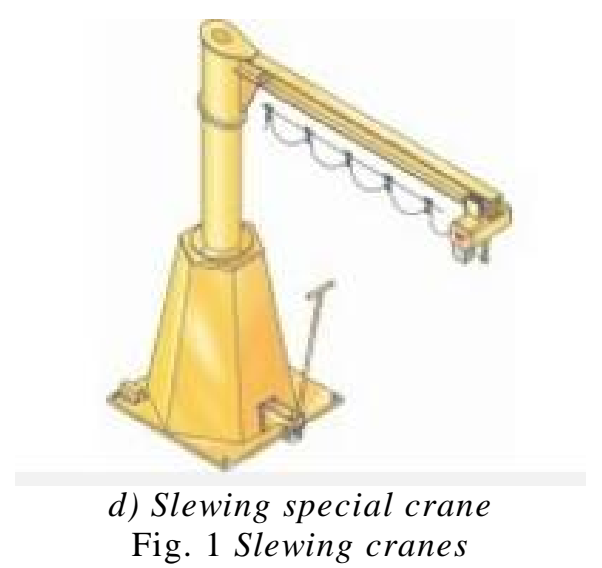

The components and role of wall crane with carriage are presented hereinafter (figure 2):

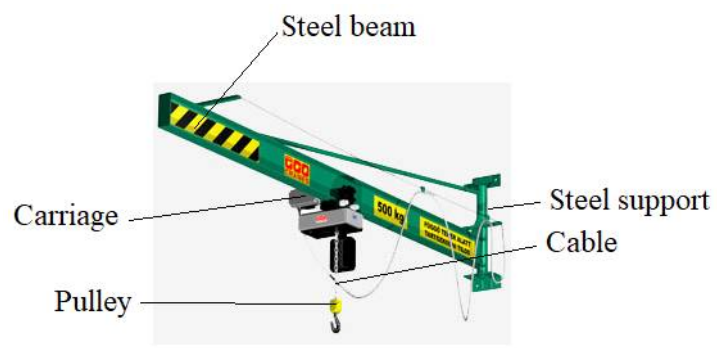

Fig. 2 Wall crane with carriage

The vertical metallic pillar is a special steel structure of U-profile which is assembled in the same conditions of the horizontal jib. The horizontal jib is a special steel structure of the "I 10" profile type. The carriage is a crane device supporting the lifting mechanism and serves for its travel on rolling way existing on the crane. Slinging and hanging of loads on the hook of cranes and lifting mechanism is done by means of slings and hanging devices specially built for this purpose (we used pulley yoke). The cable is a flexible part for movement transmission, consisting of stranded steel wire, and these are wound around a core, in more layers.

Wall cranes are provided with a rotating jib ensuring the rotation of handled load in the horizontal plane, besides lifting/lowering movement. These are performed depending on the customer's requirements. The crane is controlled by means of radio-control [5].

\section{CRANE JIB 3D MODELLING}

For wall crane modelling intended to reply to technical indexes under safe operation conditions, it is necessary to select the base material for each element of the subassemblies within crane construction.
The main technical features of the crane are the following: nominal load, $\mathrm{Q}=500 \mathrm{~kg}$; outreach, $\mathrm{R}=2,5 \mathrm{~m}$; lifting, lowering speeds, $\mathrm{V}=18 \mathrm{~m} / \mathrm{min}$; control place (from cabin or on ground); control type (electrical, pneumatic, hydraulic).

Slinging and hanging of loads on crane and lifting mechanism hooks is done by means of slings and hanging devices specially built for this purpose.

The technical prescriptions allow steel chains and cables as well as ropes and flat bars from metallic material, textolite or plastic to be used as slinging elements.

The lifting capacity of a crane or lifting mechanism means the maximum value of a mass admitted to be lifted by the crane or lifting mechanism.

The lifting capacity of jib cranes with variable outreach corresponds to the jib position for which the outreach is minimum.

The crane jib together with the carriage were designed with Mechanical Desktop 2015 software. Engineering was separately performed for the two components, meaning: jib and carriage [3], [4]. Their complete assembly is shown in figure 3 .

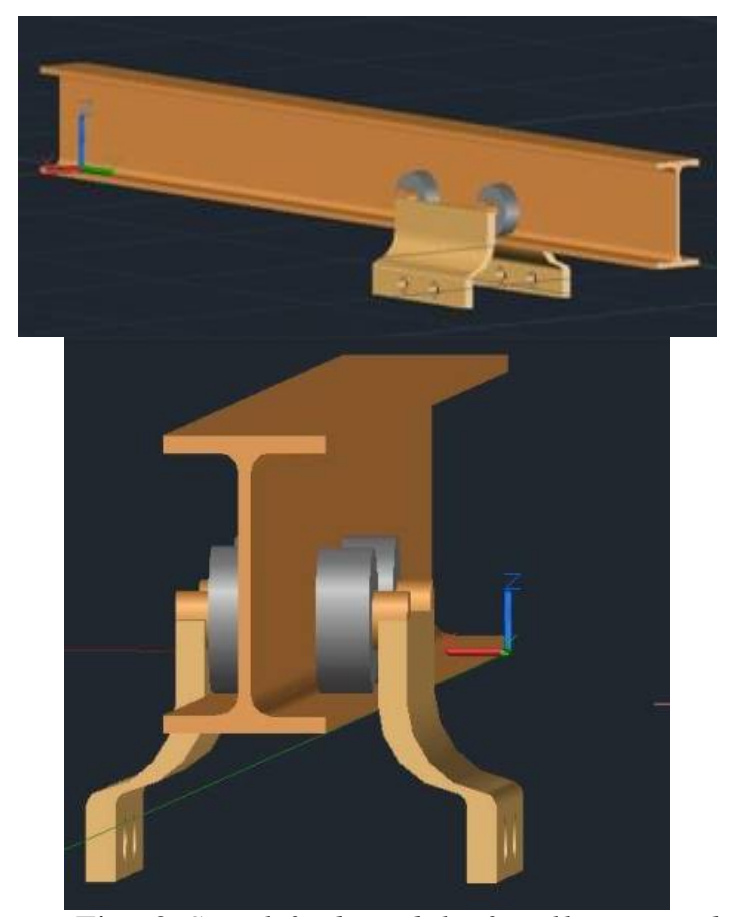

Fig. 3 Simplified model of wall crane jib with carriage

For finite elements analysis, the jib which is the most stressed component of the entire assembly was adopted. This is a construction in one piece from an "I 10" rolled profile and has the total length of 1000 $\mathrm{mm}$. 
The finite elements analysis was performed in the Algor V23 medium. The Algor V 23 analysis medium is characterized by simplicity and easiness in modelling and it offers the user complete and extremely strong instruments for analysis [2].

Taking into account the operation mode of this structural component, the adopted hypothesis for finite element analysis considers:

- $\quad$ Linear behavior of material (OL 37);

- Supporting in end joint alloys on entire inner surface, all degrees of freedom were restricted, but the rotation around $\mathrm{Y}$ axis;

The stresses are of force distributed on surface type and were so chosen to simulate the real contact between carriage rollers and rolling way;

- Due to narrow contact, we increase the contact spot to eliminate errors;

- Discretization was performed in the manual mode to be sufficiently refined taking into account the dimensions of sectional area [6], [7]. This hypothesis provides a higher trust level upon the final results.

\section{FINITE ELEMENTS ANAL YSIS OF CRANE JIB}

Analysing the obtained results, the following conclusions can be drawn:

The maximum stress condition is found in area of the two joints as can be noticed in figures below.

The stresses distribution on the free end (in the area where the stress is applied) takes into account the interaction manner between the carriage rollers and rolling way (figure 4 and figure 5).

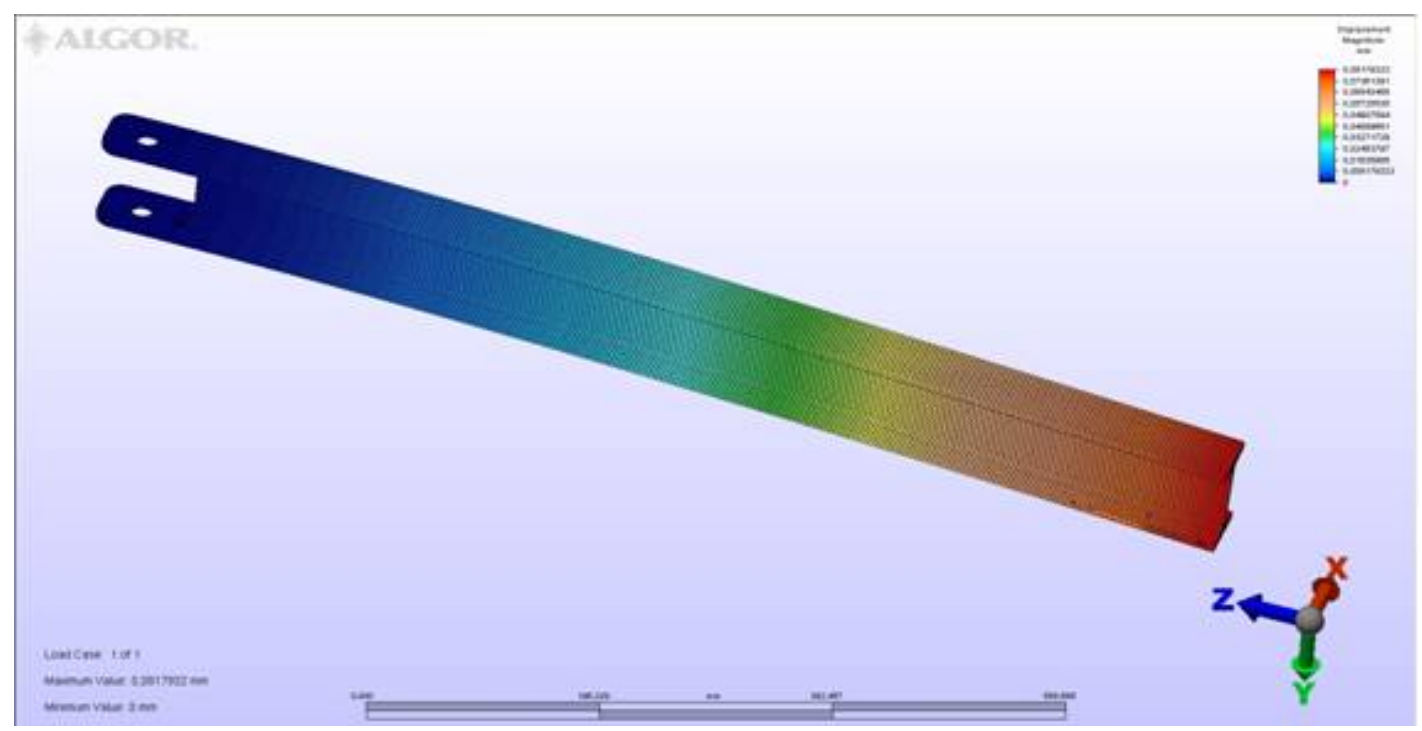

Fig. 4 A Wall crane jib analysis for offsets

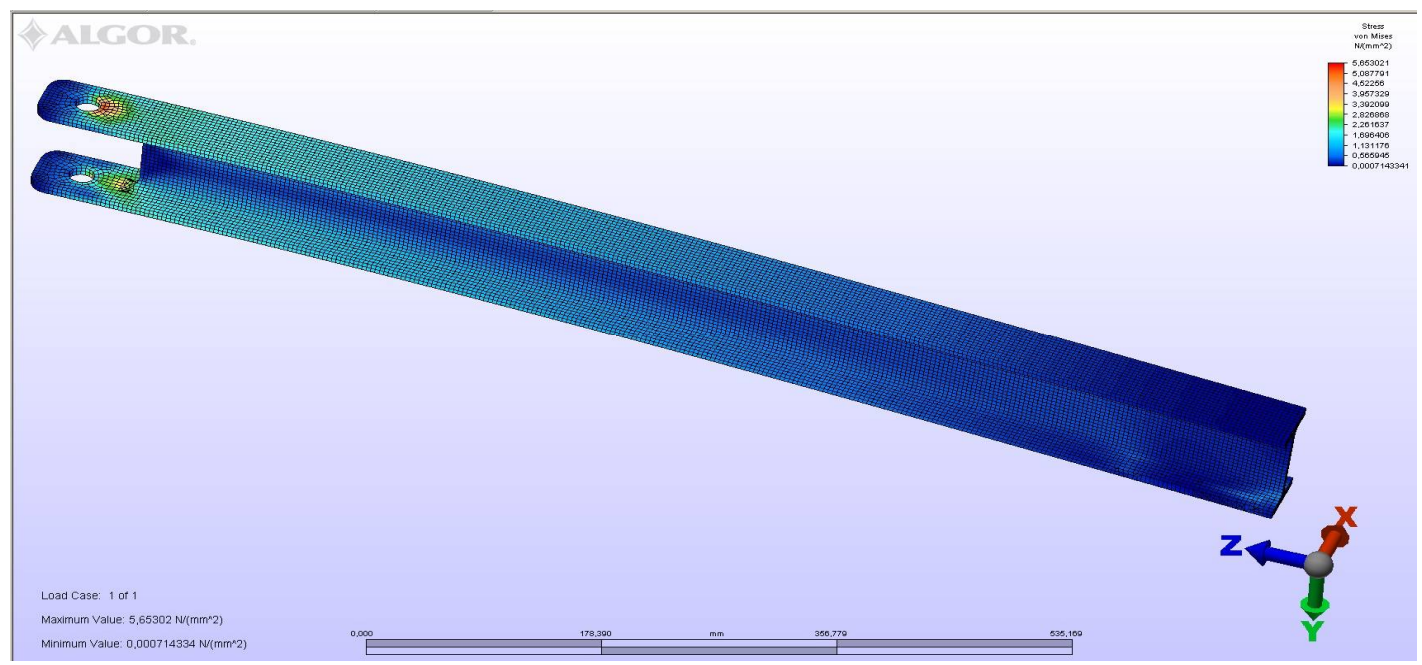

Fig. 5 Wall crane jib analysis for stresses 


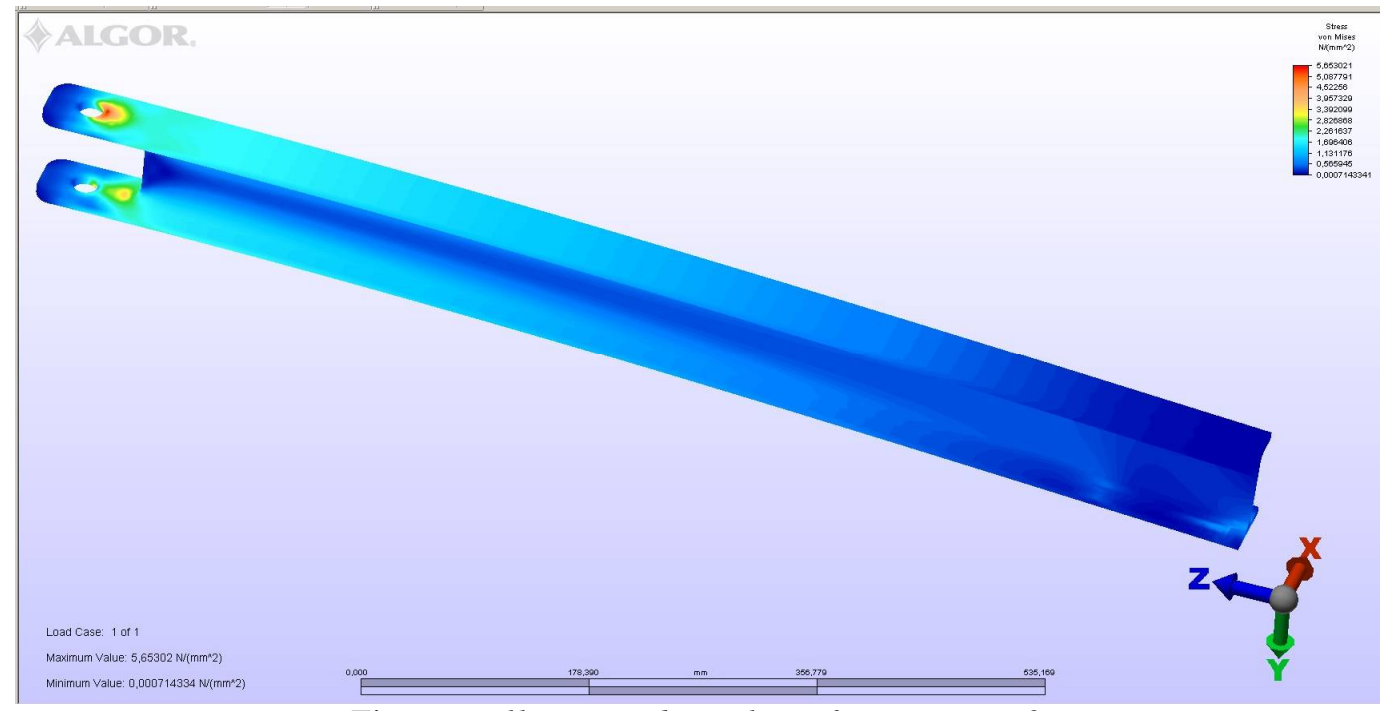

Fig. 6 Wall crane jib analysis for stresses 2

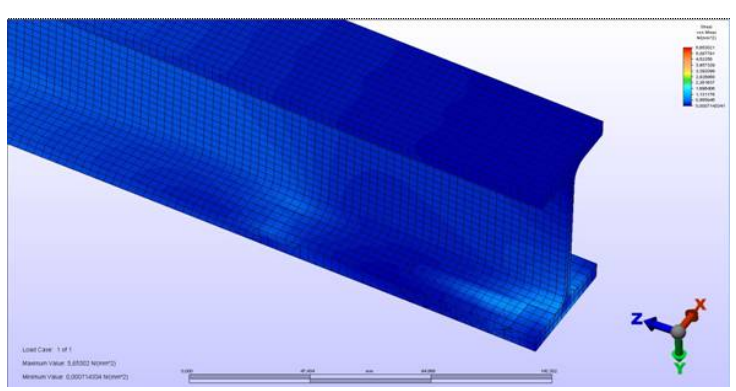

Fig. 7 Wall crane jib analysis for stresses, detail with forces

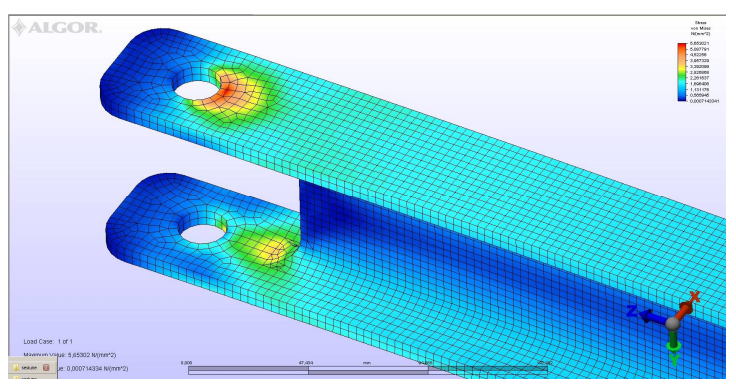

Fig. 8 Wall crane jib analysis for stresses, detail with supporting

\section{CONCLUSIONS}

The 3D parametric modelling offers some advantages in the engineering of mechanical assemblies, such as high productivity in drawing the items and assemblies once the 3D model performed workshop drawings can be extracted.

Using the finite element method in durability determination is a fast and extremely efficient method, by which we obtain a significant reduction of effort in engineering, materials and power consumption, a statement which was sustained also by research presented herein.

As expected, the maximum values of offsets were obtained on the free end, namely in the area of outer stress application.

A last conclusion refers to the fact that taking into account the adopted material, the obtained results make this item be within safe operation area, deformations being within allowed limits.

\section{REFERENCES}

[1] Alămoreanu M, Coman L, Nicolescu Ş., - “Lifting machines", Vol. 1, Technical Publishing House, Bucharest, 1996

[2] Dumitrache, P., - "Modelling of strength structures by means of finite elements methods"Impuls Publishing House, Bucharest 2003

[3] Goanță, A.M., - "Complex system of modern informatics methods for teaching graphics disciplines from technical field", International Conference on Engineering Graphics and Design, ICEG, 2009

[4] Goanță, A.M., Ghelase, D., Dașchievici, L.,"Graphic results of applying CAD tools to design automotive braking systems", Proceedings of the 9th WSEAS international conference on System science and simulation in engineering, 2010

[5] Oproescu G., "Machines and installations for industrial transport",Edmunt Publishing House, 2001, Brăila

[6] Potîrniche, A., Căpățână, G., - "Finite element analysis of an excavator bucket embedding ripper teeth", The Annals of "Dunarea de Jos" University of Galați, Fascicle XIV Mechanichal Engineering, ISSN 1224-5615, 2009

[7] Potîrniche, A., Căpățână, G., - "Computational assessements regarding modal analysis of an elevator bucket", The Annals of "Dunarea de Jos" University of Galați, Fascicle XIV Mechanichal Engineering, ISSN 1224-5615, 2009 